\title{
Determinación de la calidad bacteriológica del aire en un laboratorio de microbiología en la Universidad Distrital Francisco José de Caldas en Bogotá, Colombia
}

\author{
Bacteriological air quality in a microbiology laboratory at the Distrital Francisco \\ José de Caldas. University in Bogotá, Colombia
}

César Alberto Romero Bohórquez¹, Diego Fernando Castañeda Alvarado², Gloria Stella Acosta Peñaloza ${ }^{3}$.

\section{Resumen}

Objetivo. Determinar las bacterias del aire de un laboratorio de enseñanza de microbiología de la Universidad Distrital y así establecer la posibilidad de riesgo para la salud a la exponen los usuarios por la presencia de estos microorganismos. Métodos. Se tomaron muestras de aire por la técnica de sedimentación, se realizaron recuentos, y caracterización macroscópica y microscópica de las colonias. Después de aislamientos selectivos se llevó a cabo identificación por BD BBL Crystal. Resultados. Se obtuvo mayor recuento de bacterias Gram positivas que de Gram negativas. Se encontraron bacterias de los géneros Micrococcus, Staphylococcus, Leuconostoc, Bacillus, Corynebacterium, Pseudomonas, Yersinia, Serratia, Shigella, Klebsiella, Citrobacter y Acinetobacter. Los resultados mostraron que las bacterias identificadas no suponen riesgo elevado para la salud de los usuarios sanos, pero que es necesario implementar medidas para disminuir la carga bacteriana y disminuir posibles afecciones generales en la salud de sus ocupantes.

Palabras clave: Calidad bacteriológica del aire, calidad del aire en interiores, bacterias del aire en interiores, laboratorio de microbiología.

\section{Abstract:}

Objective. Determine airborne bacteria in a teaching laboratory of microbiology at the Distrital University and establish the bacteriological quality of air which users are exposed. Methods. Air samples were taken by sedimentation technique. The recovered colonies were counted, macroscopic and microscopic characterized and culture in selective media. The isolates were identifies by BD BBL Crystal. Results. It was found greater counts of gram-positive bacteria compared with Gram negative. It was identified bacteria of genus Micrococcus, Staphylococcus, Leuconostoc, Bacillus, Corynebacterium, Pseudomonas, Yersinia, Serratia, Shigella, Klebsiella, Citrobacter and Acinetobacter. The results showed that the bacteria identified do not present high risk to healthy of users, but it is necessary to implement measures to reduce the bacterial presence. Additionally, in this way decrease possible impact in general health of its occupants.

Key words: Bacteriological air quality, indoor air quality, indoor airborne bacteria, microbiology laboratory.

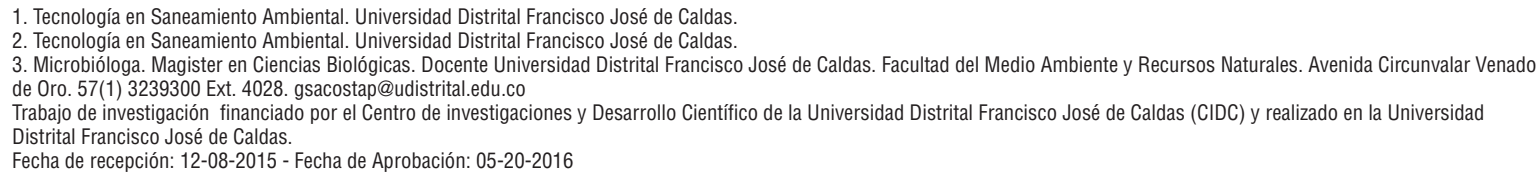




\section{Introducción}

La determinación de los microorganismos en el aire interior se vuelve importante cuando se analiza la incidencia en la salud de las personas que ocupan las edificaciones, ya que el aire es un medio de dispersión de muchos microorganismos patógenos, entre ellos las bacterias (1). Estas bacterias transportadas en el aire tienen un efecto en la calidad del aire interno de viviendas y oficinas $(2,3)$, hospitales $(2,4)$, industrias de manufactura y farmacéuticas $(5,6)$, laboratorios (7), salones de clase (8), archivos documentales $(9,10)$, museos, bibliotecas y librerías $(10)$.

Kelley y Gilbert (2) reportan Streptococcus sp., Corynebacterium sp., Flavimonas sp., Lactobacillus sp., Burkholderia sp., Bacillus sp., Bradyrhizobium sp. en ambientes de oficina y Kytococcus sedentarius, Staphylococcus epidermidis, S. haemolyticus, Ralstonia pickettii, Enterobacter sp., Kocuria rhizophila, Micrococcus luteus, Microcystis aeruginosa, Prochlorococcus marinus, Methylocella silvestris y Methylobacterium extorquens en áreas de hospitales. Castañeda-Roldán et al (5) recuperaron Escherichia sp., Staphylococcus sp., Streptococcus sp., Enterobacter sp y Bacillus sp. en el aire de una industria textil y De la Rosa et al (6) identificaron en una zona limpia de una industria farmacéutica los géneros Staphylococcus, Micrococcus, Bacillus, Corynebacterium y Arthrobacter. De las especies identificadas por Kelley y Gilbert (2) y De la Rosa et al (6), coincidieron S. epidermidis y M. luteus.

En estudios realizados dentro de universidades se han reportado diversidad de bacterias en aires interiores. Soto et al (8) llevaron a cabo un estudio en la Universidad de Murcia, en la Facultad de Biología, donde identificaron bacterias de los géneros Micrococcus, Staphylococcus, Streptococcus, Bacillus, Neisseria, Acinetobacter, Pseudomonas, Corynebacterium y Enterobacter. Estas bacterias fueron recuperadas de salones de clase, salas de lectura, corredores, cafetería, librería, baños, recepción y áreas de oficinas. Se han reportado en salones de clase bacterias de la familia Propionibacterineae, Xanthomonadaceae, Micrococcineae, Enterobacteriaceae y Corynebacterineae, y los géneros Sphingomonas, Caenibacterium, Staphylococcus; y en oficinas los géneros Streptococcus, Corynebacterium, Flavimonas, Lactobacillus, Burkholderia, Bacillus y Bradyrhizobium (2).

El interés en la calidad del aire de los laboratorios radica principalmente en los laboratorios de diagnóstico de microbiología clínica y ambientales. Por el carácter del laboratorio de microbiología clínica, en estos espacios se originan riesgos de diverso tipo, especialmente biológicos, que pueden producir problemas en la salud de los trabajadores y de los usuarios (11). En estudios realizados en laboratorios ambientales, se encuentran estudios como el realizado por Herrera et al (12) en cuatro laboratorios de instituciones públicas en la ciudad de Guatemala y Barcenas. En esta investigación se aislaron 40 géneros de bacterias, de los cuales Staphylococcus y Bacillus fueron los predominantes.

En relación con investigaciones de la calidad del aire de los laboratorios de microbiología ambiental dedicados a la enseñanza, el presente estudio es novedoso ya que no se conocen estudios en el área. El laboratorio de estudio trabaja en diferentes áreas de la microbiología relacionadas con el ambiente como microbiología básica, microbiología de alimentos, biorremediación y biotecnología.

Esencialmente, el tipo de microorganismos encontrados en el aire, tienen relación con el tipo de muestras que se trabajan, y adicionalmente con las bacterias que llegan con el viento a las edificaciones y pueden permanecer en ellas por largos periodos de tiempo influenciadas por factores ambientales como hora, temperatura, humedad, polvo y viento. Así mismo, la presencia de las bacterias depende de los propios ocupantes, los materiales inadecuados y el trabajo que se realiza en el interior (12).

Las bacterias Gram positivas predominan en los ambientes internos sobre las bacterias Gram negativas, debido a que son más resistentes y que sobreviven por más tiempo $(3,7)$ aunque 
algunas investigaciones han reportado mayor predominancia de bacterias Gram negativas (13). Soto et al (8) recuperaron en la carga microbiológica del aire interior en la Universidad de Murcia (España) especies de Micrococcus, Staphylococcus, Streptococcus, Bacillus, Neisseria, Acinetobacter, Pseudomonas, Corynetacterium y Enterobacter, donde la mayoría de las bacterias identificadas fueron cocos Gram positivos.

Herrera etal (12) identificaron en cuatro laboratorios de microbiología, los géneros Gram positivos Staphylococcus, Streptococcus, Bacillus y Micrococcus, entre otros, y los géneros Gram negativos Serratia, Salmonella, Klebsiella, Escherichia, Acinetobacter y Pseudomonas. Los laboratorios muestreados correspondieron a laboratorios de diagnóstico, ninguno a la enseñanza.

Bacterias del aire de los ambientes interiores pueden causar síntomas y enfermedades en las mucosas, en la piel, conjuntivitis y dolor de cabeza, y algunas otras pueden producir enfermedades respiratorias (12-13). Es así que, Streptococcus pyogenes produce amigdalitis, faringitis y bronquitis, Streptococcus pneumoniae, Staphylococccus aureus y Klebsiella pneumoniae son causantes de neumonía clásica, y Neisseria meningitidis es agente causal de meningitis (1). También se encuentran en el aire bacterias patógenas oportunistas que pueden producir enfermedades graves en personas susceptibles por su estado de salud (14).

Por otro lado, muchas bacterias permanecen en los espacios cuando no se cumplen los protocolos de bioseguridad en los laboratorios. Es importante tener en cuenta que algunas bacterias presentes en el aire interno de los edificios no son relevantes para la salud humana, ya que los microorganismos son un componente normal de los ambientes.

En el laboratorio de microbiología objetivo del estudio se llevan a cabo trabajos académicos de clases y de investigación. Este laboratorio que presta servicio académico a un número alto de estudiantes de programas de áreas ambientales, podría contener bacterias en el aire que ocasionan efectos sobre la salud de las personas que lo ocupan. Puesto que no se cuenta con un estudio de microorganismos en el aire de este laboratorio, se planteó esta investigación que tuvo como objetivo la determinación de las bacterias del aire del laboratorio y así establecer la posibilidad de riesgo para la salud a la exponen los usuarios por la presencia de estos microorganismos.

\section{Materiales y métodos.}

La toma de muestras para recuperación de bacterias en el aire se realizó en el Laboratorio de Microbiología de la Facultad del Medio Ambiente y Recursos Naturales de la Universidad Distrital Francisco José de Caldas en Bogotá, Colombia. El laboratorio de interés para esta investigación es utilizado para enseñanza y se realizan adicionalmente algunas actividades de investigación de los estudiantes. En este laboratorio se imparten clases de microbiología general, manejo e higiene de alimentos, biorremediación y biotecnología.

Los procedimientos de preparación de medios de cultivo y las siembras para la obtención de las colonias puras e identificación de las bacterias fueron realizados en cabina de flujo laminar para garantizar confiabilidad en la recuperación de las bacterias del aire. Se llevaron a cabo controles de esterilidad de medios de cultivo y del ambiente de la cámara de flujo laminar para asegurar confiabilidad del trabajo realizado.

\section{Muestreo del aire.}

Los puntos de muestreo se seleccionaron en base a estudios preliminares en el laboratorio donde se recuperaron las mayores concentraciones de bacterias en el aire. El primer punto de muestreo (M) correspondió a un mesón de trabajo de los estudiantes (altura $0.92 \mathrm{~m}$ ) y el segundo punto (A) a un mueble de almacenamiento de materiales (altura $2.0 \mathrm{~m}$ ). En total se llevaron a cabo 10 momentos de toma de muestra de aire para un total de 20 muestras, entre la última semana de octubre y la primera semana de diciembre de 2014 . 
Las muestras de aire se recolectaron en diferentes horas del día, durante actividades académicas diferentes, con el fin de recuperar las bacterias en las diferentes condiciones generadas en este espacio. El laboratorio funciona de la 6:00 a.m. a las 10:00 p.m. de lunes a viernes y tiene una capacidad para 20 estudiantes.

Para la recuperación de las bacterias del aire se utilizó la técnica de sedimentación por gravedad (1), exponiendo cajas de Petri (90 $\mathrm{mm}$ de diámetro) con agar nutritivo (AN) abiertas por 15 minutos. Se utilizó AN como medio no selectivo para el recuento y aislamiento de las bacterias $(8,15)$. Las cajas fueron incubadas a $37^{\circ} \mathrm{C}$ por 48 horas, para la recuperación de bacterias mesófilas aerobias (13).

Recuento, aislamiento e identificación de las bacterias.

Al término de la incubación se realizó el conteo de la colonias y se informaron como Unidades Formadoras de Colonia (UFC) por $\mathrm{m}^{3}$ de aire de acuerdo a la fórmula propuesta por Omeliansky (16):

$$
\mathrm{N}=5 \mathrm{a} \times 10^{4}(\mathrm{bt})^{-1}
$$

Donde $\mathrm{N}$ es el número de microorganismos en el aire interno, a es el número de colonias en la caja de petri, b es el superficie de la caja $\left(\mathrm{cm}^{2}\right)$, t es el tiempo de exposición (minutos).

A partir de las bacterias recuperadas por la técnica de sedimentación en AN se obtuvieron colonias puras por siembras sucesivas por rejilla en $\mathrm{AN}, 37^{\circ} \mathrm{C}$ por 48 horas. Las cepas puras obtenidas fueron caracterizadas macroscópicamente y se caracterizaron microscópicamente mediante coloración de Gram (17). A partir de estos resultados se determinó el predominio de las bacterias según la coloración de Gram (12).

De acuerdo con el objetivo de determinar las bacterias asociadas a posibles afecciones a la salud, las colonias fueron re-sembradas en agares selectivos; agar Eosina Azul de Metileno, agar Cetrimide, agar Brillance Bacillus Cereus y Agar Baird Parker, y se incubaron a $37^{\circ} \mathrm{C}$ por 48 horas. A partir de las recuperaciones en los agares selectivos, la morfología macroscópica diferencial y la confirmación del Gram se seleccionaron los morfotipos para identificación.

Para la identificación, las bacterias seleccionadas fueron sembradas en agar sangre para obtener un cultivo fresco, $24 \mathrm{~h}$ a $37^{\circ} \mathrm{C}$, y fueron identificadas por el método BD BBL Crystal (18). Se realizaron pruebas bioquímicas a las bacterias con un valor de confianza bajo $(<0.80)$ para confirmación de la identificación.

\section{Resultados}

Los recuentos obtenidos en los puntos de muestreo mostraron en promedio mayor número de bacterias $\left(655 \mathrm{UFC} / \mathrm{m}^{3}\right)$ en el punto A que en el punto B (271 $\mathrm{UFC} / \mathrm{m}^{3}$ ) evidenciándose mayor concentración bacteriana a una altura de $0.92 \mathrm{~m}$ en el muestreo en el mesón de estudiantes, que a una altura mayor en el estante de almacenamiento (2.0m), Tabla 1.

Tabla 1. Concentración de bacterias en el aire por punto de muestreo $\left(\mathrm{UFC} / \mathrm{m}^{3}\right)$

\begin{tabular}{ccc}
\hline Muestreo & M $(\mathbf{0 . 9 2} \mathbf{m})$ & $\mathbf{A}(\mathbf{2 . 0} \mathbf{~})$ \\
\hline 1 & 419.2 & 104.8 \\
2 & 1152.8 & 366.8 \\
3 & 681.2 & 52.4 \\
4 & 681.2 & 0 \\
5 & 1048 & 733.6 \\
6 & 471.6 & 366.8 \\
7 & 838.4 & 681.2 \\
8 & 786 & 157.2 \\
9 & 209.6 & 157.2 \\
\hline 10 & 262 & 104.8 \\
\hline Promedio & 655 & 272.5 \\
\hline
\end{tabular}

M: Mesa A: Armario Fuente: Los autores

La caracterización microscópica por la coloración de Gram, de las bacterias recuperadas en AN, mostraron mayor cantidad de bacterias Gram positivas $(77.4 \%)$ que de bacterias Gram negativas (22.6\%), Tabla 2. 
Tabla 2. Número de bacterias recuperadas del aire durante el estudio.

\begin{tabular}{cccc}
\hline Lugar de muestra & Gram positivas & Gram negativas & Total \\
\hline M (0,92m) & 94 & 31 & 125 \\
A (2,0 m) & 43 & 9 & 52 \\
Total & 137 & 40 & 177 \\
\hline
\end{tabular}

M: Mesa A: Armario Fuente: Los autores

De las bacterias que crecieron en los medios macroscópica y microscópica, 30 bacterias fueron selectivos, que se seleccionaron por morfología identificadas mediante BD BBL Crystal, Tabla 3.

Tabla 3. Bacterias recuperadas e identificadas en el aire del laboratorio.

\begin{tabular}{|c|c|c|c|}
\hline Gram & Microorganismo & $\begin{array}{c}\text { Valor de Confianza } \\
\text { BBL Crystal } \\
\end{array}$ & $\begin{array}{c}\text { Número de colonias } \\
\text { identificadas }\end{array}$ \\
\hline \multirow{10}{*}{ Gram Positivas } & Micrococcus sedentarius & 0.99 & 9 \\
\hline & Micrococcus sedentarius & $0.46^{*}$ & 1 \\
\hline & Staphylococcus haemolyticus & 0.93 & 2 \\
\hline & Staphylococcus capitis & 0.99 & 2 \\
\hline & Staphylococcus cohnii sp. cohnii & 0.86 & 1 \\
\hline & Staphylococcus hominis & 0.99 & 1 \\
\hline & Staphylococcus lentus & 0.99 & 1 \\
\hline & Leuconostoc pseudomesenteroides & 0.92 & 1 \\
\hline & Bacillus subtilis & 0.99 & 1 \\
\hline & Corynebacterium renale Group & 0.99 & 1 \\
\hline \multirow{9}{*}{ Gram negativas } & Pseudomonas stutzeri & 0.98 & 1 \\
\hline & Pseudomonas putida & 0.95 & 1 \\
\hline & Yersinia pseudotuberculosis & 0.92 & 1 \\
\hline & Yersinia enterocolitica Group. & $0.51^{*}$ & 1 \\
\hline & Serratia fonticola & $0.74^{*}$ & 1 \\
\hline & Shigella sp. & 0.99 & 1 \\
\hline & Klebsiella pneumoniae sp. ozaenae & 0.96 & 1 \\
\hline & Citrobacter freundii & 0.91 & 1 \\
\hline & Acinetobacter iwoffii & 0.99 & 2 \\
\hline
\end{tabular}

* Confirmación mediante pruebas bioquímicas.

Fuente: Los autores.

Entrelabacterias Gram positivas, 10 colonias fueron identificadas como Micrococcus, específicamente M. Sedentarius. Se identificaron cinco especies de Staphylococcus, una de Leuconostoc, una de Bacillus y una de Corynebacterium.
Entre las bacterias Gram negativas se identificaron dos especies de Pseudomonas, dos especies de Yersinia, una de Serrlatia, una de Shigella, una de Klebsiella, una de Citrobacter y una de Acinetobacter. 


\section{Discusión}

El ambiente interno de los edificios es un hábitat natural para los microorganismos cuyas fuentes incluyen las entradas de aire por puertas y ventanas, las actividades que se realizan en el espacio, y las personas directamente a través de la piel y la respiración. Estos microorganismos pueden permanecer inactivos por un tiempo en el polvo $y$ en las superficies, $y$ en algunas circunstancias pueden ser metabólicamente activos como poblaciones proliferantes (3).

En el laboratorio de microbiología de la Universidad Distrital, la mayor cantidad de bacterias recuperadas en el punto de muestreo en la mesa (M) coincide con lo afirmado por De la Rosa et al (1) que el crecimiento microbiano es mayor cerca del suelo, sobre todo en los dos metros inferiores. También esto se relaciona con que en esta zona se ubican las personas y por lo tanto son reflejo del microclima del hombre (13).

Estudios realizados en salones de clase y laboratorios muestran cargas variadas de bacterias en ambientes interiores. Las concentraciones de bacterias obtenidas del aire fueron de 198.33 $\mathrm{UFC} / \mathrm{m}^{3}$ a $347.5 \mathrm{UFC} / \mathrm{m}^{3}$ en un estudio realizado en salones de una escuela de secundaria en Jodhpur, Rajasthan (India) (13). Herrera et al (12) determinaron cargas entre $220 \mathrm{UFC} / \mathrm{m}^{3}$ y de 790 $\mathrm{UFC} / \mathrm{m}^{3}$ en el ambiente interno del Laboratorio de la Autoridad en el Manejo Sustentable del Lago de Amatitlán en Bárcenas. En ciudad de Guatemala, determinaron cargas bacterianas entre $130 \mathrm{UFC/}$ $\mathrm{m}^{3}$ y $660 \mathrm{UFC} / \mathrm{m}^{3}$ en el laboratorio de la Empresa Municipal de Agua, entre $80 \mathrm{UFC} / \mathrm{m}^{3}$ y $250 \mathrm{UFC/}$ $\mathrm{m}^{3}$ en el Laboratorio de Análisis Fisicoquímico y Microbiológico, y entre 50 y $360 \mathrm{UFC} / \mathrm{m}^{3}$ en el Laboratorio Microbiológico de Referencia. Aunque en el estudio no se encontraron niveles altos de incidencia en la salud del personal estable en los cuatro laboratorios muestreados, los autores verificaron influencia de la falta de procedimientos de limpieza, desinfección adecuada y ventilación sobre la calidad del aire de cada laboratorio. García et al (7) realizaron un estudio en tres laboratorios de la Facultad de Ingeniería de la Universidad del Zulia en Venezuela, Laboratorio de Carbón, Centro de Estudios de Corrosión y Laboratorio de Tecnología de Alimentos y Fermentaciones, estimulados por el manejo de bacterias en estos espacios y su posible efecto en las personas que permanecen en ellos. En todas las áreas muestreadas el nivel promedio de microorganismos superó $10^{4} \mathrm{UFC} / \mathrm{m}^{3}$, con los recuentos mayores en el Laboratorio de Carbón que adicionalmente reportó los valores más altos de polvo total y polvo respirable.

En el laboratorio de microbiología de la Universidad Distrital, la mayor carga bacteriana fue de 1152,8 $\mathrm{UFC} / \mathrm{m}^{3}$ en el punto de muestreo del mesón de estudiantes (M) y de $681,2 \mathrm{UFC} / \mathrm{m}^{3}$ en el punto de muestreo del mueble de almacenamiento (A), y el promedio de bacterias en los dos puntos seleccionados fue de $463,6 \mathrm{UFC} / \mathrm{m}^{3}$. Es importante resaltar, que el laboratorio de microbiología objeto del estudio es un espacio dedicado a la enseńanza, con un alto flujo de estudiantes durante todo el día, 5 días a la semana, lo que podría explicar recuentos mayores a los encontrados por Herrera $e t$ al (12). Así mismo en este laboratorio se verifican problemas en procedimientos de limpieza y ventilación, principalmente. Es así que al comparar los recuentos obtenidos en el laboratorio de microbiología de la Universidad Distrital, con otros estudios, se evidencia que hay que revaluar procesos de limpieza, desinfección y ventilación, ya que los recuentos bacterianos obtenidos son indicadores de la calidad microbiana en ambientes internos y de las prácticas de higiene llevadas a cabo en ellos.

Como se ha discutido anteriormente, los recuentos de bacterias en el aire interior de las edificaciones es importante para determinar la calidad del aire. Así mismo, es importante la determinación de las bacterias que predominan. Soto et al (8) que estudiaron el aire interno de la Universidad de Murcia encontraron predominancia de las bacterias Gram positivas (85\%) de los géneros Micrococcus, Staphylococcus y Streptococcus en su mayoría, y en menor proporción Bacillus y 
Corynebacterium. Herrera et al (12) encontraron predominancia de Staphylococcus y Bacillus, seguido por Celullomonas en un estudio de impacto de la calidad microbiológica del aire externo sobre el aire interno de cuatro laboratorios de microbiología en Guatemala. Aunque la mayoría de las bacterias Gram positivas aisladas en ambientes interiores son microflora saprófita, generalmente se asocian a la piel y a las mucosas, y por tanto, son resultado de la presencia de personas. Sin embargo, algunos géneros Gram positivos afectan el tracto respiratorio superior e inferior, produciendo enfermedades como faringitis y bronquitis, respectivamente (1). Adicionalmente, su presencia puede indicar deficiencias en los sistemas de ventilación (8). Las bacterias Gram negativas son más sensibles que las Gram positivas a las condiciones ambientales y permanecen por tiempos más cortos (1). Estas bacterias producen endotoxinas compuestas por lipopolisacáridos de la pared celular que pueden ser inhaladas con el polvo de los ambientes internos. Estas endotoxinas pueden producir efectos tóxicos, produciendo efectos perjudiciales como fiebre y problemas cardiovasculares, bronquitis y asma por la exposición continua a estas bacterias (13). Es así, que la predominancia de bacterias Gram positivas en el laboratorio de microbiología de la Universidad Distrital podría estar determinada por la presencia de polvo y de las actividades de las personas (13).

En el aire interno del laboratorio, entre las bacterias Gram positivas se aislaron especies de Micrococcus, Streptococcus, Staphylococcus, Bacillus y Corynebacterium, bacterias aisladas en estudios en ambientes internos por otros investigadores. Rintala et al (19) determinaron predominancia de Corynebacterium sp., Propionibacterium sp., Staphylococcus sp. y Streptococcus sp. en el polvo del aire interior de dos edificios. Borrego et al (9) aislaron estos géneros en dos depósitos del Archivo Nacional de la República de Cuba y adicionalmente aislaron bacterias Gram negativas, entre las que se nombra Serratia marcenscens. En un estudio en cuatro museos, dos librerías y dos archivos en Polonia, Skóra et al (10) aislaron $B$. subtilis en el aire y superficies en dos museos, un archivo y una librería, $S$. lentus en un museo, un archivo y las dos librerías, $S$. cohnii en un archivo, y $S$. haemolyticus y $S$. hominis en un archivo. En el estudio de Skóra et al (10) no se reporta aislamiento de bacterias Gram negativas.

Los géneros Staphylococcus y Streptococcus pueden relacionarse con procesos inflamatorios por penetración a través de la piel e ingreso a las vías respiratorias y Serratia sp. ha sido relacionada con enfermedades respiratorias (9). Por otro lado, el género Micrococcus hace parte de la microflora cutánea que ha sido aislado del aire y del suelo (20). Así mismo, los géneros Streptococcus y Corynebacterium hacen parte de la flora normal de la piel (21).

Encuantoalas bacterias Gramnegativas, lasbacterias pertenecientes a las familia Enterobacteriaceae están también relacionadas con la presencia de los humanos en los ambientes internos y se han encontrado por ingreso desde ambientes externos (21). Este mismo comportamiento se ha determinado para especies de Acinetobacter y Pseudomonas que fueron aisladas de ambientes internos en la Universidad de Murcia, en Espańa, del aire en zonas de la Facultad de Biología (8), géneros que fueron aislados por Toloza-Moreno et al (13) en el archivo central de la Universidad Pedagógica y Tecnológica de Colombia. Por otro lado, Herrera et al (12) aislaron en laboratorios de microbiología en Guatemala, Pseudomonas putida y Klebsiella pneumoniae, como ocurrió en este estudio en el laboratorio de microbiología de la Universidad Distrital Francisco José de Caldas.

De las bacterias aisladas en el laboratorio, de acuerdo a la clasificación de agentes biológicos de la Organización Mundial de la Salud (OMS), las que pueden causar enfermedad en el hombre y pueden suponer un peligro para los trabajadores, siendo poco probable que se propague a la colectividad se encuentran Corynebacterium renale, Klebsiella pneumoniae sp. ozaenae, Yersinia enterocolitica y Yersinia pseudotuberculosis. Estas bacterias están clasificadas en el grupo de riesgo 
2 (riesgo individual moderado, riesgo poblacional bajo) que incluye los agentes patógenos que pueden provocar enfermedades en humanos pero que tienen pocas probabilidades de entrañar un riesgo grave para el personal del laboratorio (22).

Las otras bacterias identificadas son clasificadas por la OMS en el grupo agente biológico del grupo 1 (riesgo individual y poblacional escaso o nulo) que corresponde a aquel que resulta poco probable que cause una enfermedad en el hombre (23-29). Esta clasificación de la OMS (22) por grupos de riesgo se utiliza exclusivamente para el trabajo de laboratorio y supone un peligro relativo. El laboratorio de microbiología de la Facultad del Medio Ambiente y Recursos Naturales de la Universidad Distrital se clasifica en el nivel básico de bioseguridad 1, enseñanza básica e investigación, donde solo se requiere de equipo de seguridad, y el trabajo se lleva a cabo en mesa de laboratorio al descubierto (21). La presencia de las bacterias en el aire indica su manejo en la prácticas académicas, en investigación o su introducción a través de las personas, o del aire exterior.

De acuerdo con lo anterior las bacterias aisladas e identificadas no suponen un peligro para las personas en el laboratorio, ya que ninguna se encuentra en los grupos de riesgo 3 y 4 (22). Sin embargo, aunque la mayoría de las bacterias recuperadas del laboratorio están clasificadas en el grupo de riesgo individual y poblacional escaso o nulo, hay que tener en cuenta que se recuperaron cuatro especies clasificadas en el grupo de riesgo individual moderado, riesgo poblacional bajo. Por tal razón, el laboratorio debe implementar medidas de limpieza, desinfección y ventilación adecuadas para disminuir las poblaciones de bacterias en el aire, y disminuir posibles afecciones generales en la salud de sus ocupantes.

\section{Agradecimientos}

Se agradece al Centro de Investigaciones y Desarrollo Científico de la Universidad Distrital
Francisco José de Caldas por el apoyo brindado para la realización del presente trabajo.

\section{Referencias}

1. De la Rosa M del C, Mosso MA, Ullán C. El aire: Hábitat y medio de transmisión de microorganismos. Obs. Medioambient. 2002; 5:375-402.

2. Kelley ST, Gilbert JA. Studying the microbiology of the indoor environment. Genome Biology. 2013;14(2):202. Disponible en: http://www.genomebiology.com/content/pdf/gb-2013-14-2-202.pdf

3. Konya T, Scott J. Recent advanced in the microbiology of the built environment. Curr Sustainable Renewable Energy Rep. 2014;1(2):35-42. Disponible en: http://link.springer.com/ article/10.1007/s40518-014-0007-4

4. Cifuentes Y, Ruiz A, Leal A, Muñoz L, Herrera M, Jiménez L. Perfil microbiológico de aislamientos en unidades neonatales en un hospital de tercer nivel de Bogotá, Colombia. Rev. Salud pública. 2005;7(2):191-200. Disponible en: http://www. scielo.unal.edu.co/scielo.php?script=sci_arttext\&pid=S012400642005000200007\&lng=es

5. Castañeda-Roldán E, Rivera-Tapia JA, Lechuga-Batista, K. Determinación de la calidad microbiológica del aire en una industria textil. Rev Latinam Salud Trab. 2003;3(1):21-24.

6. De la Rosa M del C, Ullán C, Prieto MP, Mosso MA. Calidad microbiológica del aire de una zona limpia en una industria farmacéutica. Anal. Real Acad. Farm. 2000;66:1-17.

7. García N, Araujo I, Fernández M, Salcedo W, Cárdenas C, Fernández J et al. Calidad microbiológica y fisicoquímica del aire en tres laboratorios de la Facultad de Ingeniería de la Universidad del Zulia. Ciencia. 2005;13(2):182-192.

8. Soto T, García-Murcia RM, Franco A, Vicente-Soler J, Cansado J, Gacto M. Indoor airborne microbial load in a spanish university (University of Murcia, Spain). Anales de biología. 2009;31:109-115. Disponible en: http://dialnet. unirioja.es/servlet/articulo? codigo $=3251124$

9. Borrego S, Pons V, Perdomo, I. La contaminación microbiana del aire en dos depósitos del Archivo Nacional de la República de Cuba. Revista CENIC Ciencias Biológicas. 2008;39(1):63-69.

10. Skóra J, Gutarowska B, Pielech-Przybylska K, Stępień Ł, Pietrzak K, Piotrowska M et al. Assessment of microbiological contamination in the work environments of museums, archives and libraries. Aerobiologia. 2015; 31(3):389-401. Disponible en: http://link.springer.com/article/10.1007/ s10453-015-9372-8

11. Rojo-Molinero E, Alados JC, Gómez de la Pedrosa E, Leiva J, Pérez J. L. Seguridad en el laboratorio de Microbiología Clínica. Enferm Infecc Microbiol Clin. 2015; 33(6):404-410. Disponible en: http://www.sciencedirect.com/science/article/ pii/S0213005X14002882

12. Herrera K, Cóbar O, De León J, Rodas A, Boburg S, Quan $\mathrm{J}$ et al. Impacto de la calidad microbiológica del aire externo en el ambiente interno de cuatro laboratorios de instituciones 
públicas en la ciudad de Guatemala y Bárcenas, Villa Nueva. Revista Científica. 2012;22(1):30-38

13. Toloza-Moreno DL, Lizarazo-Forero LM, Blanco-Valvuena, JO. Concentración y composición microbiana en el ambiente de la biblioteca central Jorge Palacios Preciado de la Universidad Pedagógica y Tecnológica de Colombia, Tunja, Colombia. Actual Biol. 2012;34(97):241-252.

14. Carazo-Fernández L, Fernández-Álvarez R, González-Barcala FJ, Rodríguez-Portal JA. Indoor air contaminants and their impact in respiratory pathologies. Arch Bronconeumol. 2013;49(1):22-27. Disponible en: http://www.sciencedirect. com/science/article/pii/S1579212912002108.

15. Naruka K, Gaur J. Microbial air contamination in a school. Int J Curr Microbiol App Sci. 2012;2(12):404-410. http:// www.ijcmas.com/vol-2-12/Kavita $\% 20$ Naruka $\% 20$ and $\% 20$ Jyoti\%20Gaur.pdf

16. Bogomolova E, Kirtsideli I. (2009). Airborne fungi in four stations of the St. Petersburg Underground rail system. International Biodet Biodegr. 63, 156-160. Disponible en: http://www. sciencedirect.com/science/article/pii/S0964830508001510

17. Maldonado-Vega M, Peña-Cabriales JJ, De Los SantosVillalobos S, Castellanos-Arévalo A P, Camarena-Pozos D, Arévalo-Rivas B et al. Bioaerosoles y evaluación de la calidad del aire en dos centros hospitalarios ubicados en León, Guanajuato, México. Rev. Int. Contam. Ambient. 2014;30(4):351-363. Disponible en: http://www.scielo.org. mx/scielo.php?pid=S018849992014000400004\&script=sci_arttext

18. Corrales-Ramírez LC, Sánchez-Leal LC, Escucha-Rodríguez FA. Determinación de la presencia de bacterias patógenas para el humano en aguas de riego en la cuenca alta de la sabana de Bogotá; D.C. Colombia. NOVA. 2014; 12(21):179186. Disponible en: http://www.unicolmayor.edu.co/ publicaciones/index.php/nova/article/view/277

19. Rintala H, Pitkäranta M, Toivola M, Paulin L, Nevaleinen A. Diversity and seasonal dynamics of bacterial community in indoor environment. BMC Microbiology. 2008; 8:56. Disponible en : http://link.springer.com/ article/10.1186\%2F1471-2180-8-56
20. Kocur M, Kloos WE, Schleiter KH. The genus Micrococcus. The prokariotes. 2006;3:961-971.

21. Adams RI, Bateman AC, Bik HM, Meadow JF. Microbiota of the indoor environment: a meta-analysis. Microbiome. 2015;3: 49. Disponible en: http://link.springer.com/article/10.1186/ s40168-015-0108-3/fulltext.html

22. Organización Mundial de la Salud (OMS). Manual de bioseguridad en el laboratorio. Organización Mundial de la Salud. Tercera edición. Ginebra. 2005. Disponible en: http:// www.who.int/csr/resources/publications/biosafety/CDS_ CSR_LYO_2004_11SP.pdf

23. Instituto Nacional de Seguridad e Higiene en el Trabajo. Guía técnica para la evaluación y prevención de los riesgos relacionados con la exposición a agentes biológicos. INSHT. Madrid. 2014. Disponible en: http://www.insht.es/InshtWeb/ Contenidos/Normativa/GuiasTecnicas/Ficheros/agen_bio.pdf

24. De Navia SLÁ, Torres SME. Calidad sanitaria del agua del Parque Natural Chicaque. Nova. 2013;11(20).

25. Nausa JG. Evaluación Clínica y radiográfica de injertos biocerámicos tipo Hidroxiapatita como alternativa en la reconstrucción de alveolos dentarios postexodoncia. Nova. 2014;12(22).

26. Galvez ZYA, Burbano VEM. Bacillus: género bacteriano que demuestra ser un importante solubilizador de fosfato. NOVA Publicación en Ciencias Biomédicas. 2015;12(22):165-77.

27. Ramírez LCC, Leal LCS, Rodríguez FAE. Determinación de la presencia de bacterias patógenas para el humano en aguas de riego en la cuenca alta de la sabana de Bogotá; DC Colombia. Nova. 2014;12(22).

28. Campuzano S, Mejía Flórez D, Madero Ibarra C, Pabón Sánchez P. Determinación de la calidad microbiológica y sanitaria de alimentos preparados vendidos en la vía pública de la ciudad de Bogotá DC. Nova. 2015;13(23):81-92.

29. Corrales LC, Romero DMA, Macías JAB, Vargas AMC. Bacterias anaerobias: procesos que realizan y contribuyen a la sostenibilidad de la vida en el planeta. Nova. 2015;13(24):5582. 